\title{
Supporting information: Predicting lithium iron oxysulfides for battery cathodes
}

\author{
Bonan Zhu ${ }^{*, \dagger, \ddagger}$ and David O. Scanlon ${ }^{*, \dagger, 9, \S, \ddagger}$ \\ $\dagger$ †epartment of Chemistry, University College London, 20 Gordon St, Bloomsbury, London \\ WC1H OAJ, United Kingdom \\ $\ddagger$ The Faraday Institution, Quad One, Becquerel Avenue, Harwell Campus, Didcot, OX11 \\ ORA, United Kingdom \\ IThomas Young Centre, University College London, Gower Street, London WC1E 6BT, \\ United Kingdom \\ $\S$ Diamond Light Source Ltd., Diamond House, Harwell Science and Innovation Campus, \\ Didcot, Oxfordshire OX11 ODE, UK \\ E-mail: bonan.zhu@ucl.ac.uk; d.scanlon@ucl.ac.uk
}

\section{Formation energies from DFT calculations}

Formation energies calculated using standard density functional theory are known to contain significant systemic errors. For example, those of many binary oxides are under-predicted due to the over-binding of molecular oxygen (using local/semi local functionals), ${ }^{1 / 3}$ which can be corrected by applying a rigid energy shift. For transition metal (TM) oxides, additional errors arise from the localized d-electrons, and often the Hubbard correction is required. One way for obtaining the desirable $U$ values is by fitting the formation energies ${ }^{4}$ of TM oxides to those obtained experimentally. Still, energies of GGA+U calculations cannot be mixed with those from standard DFT. This can be overcome by applying another rigid energy shift 
$\left(\Delta E_{M}\right)$ to the GGA+U energies. ${ }^{5}$ The two corrections mentioned above are used by the Materials Project ${ }^{[6]}$ where the Hubbard correction is only applied to oxides and fluorides of transition metals, and standard GGA calculations are used for sulfides. A slightly different approach is used by the Open Quantum Materials Database, ${ }^{7}$ where the GGA+U is used for all materials containing the transition metals, and the elemental energies are adjusted to obtain the best fit with the experimental formation energies.

In this work, we are mainly concerned about the stability of oxysulfides, where the competing phases are binary/ternary oxides and sulfides. We apply the Hubbard correction to the oxysulfides because Fe ions are expected to be in the +2 or +3 oxidation states, and it is also applied for sulfides. In contrast, the Materials Project's approach computes the competing $\mathrm{FeS}$ and $\mathrm{FeS}_{2}$ phases using standard GGA. To evaluate the schemes, the reaction energies of several reactions are computed and compared with the values obtained using experimental formation energies obtained from the Kubaschewski Table. q The differences between the theoretical and the experimental values are shown in Figure S1, where All refers to the scheme where GGA+U is used for all species, and Oxide only is calculated using GGA for sulfides, and with the $\Delta E_{M}$ term applied to the oxides treated with GGA+U. The $M P-$ ORIG refers to the data extracted directly from the Materials Project with their corrections applied. Note that the $U_{\text {eff }}(4.0 \mathrm{eV})$ and $\Delta E_{M}(1.787 \mathrm{eV})$ terms in All and Oxide only are refitted using the methodologies that MP-ORIG refers to, 415 and we are not able to reproduce the fitted values for the latter $\left(\mathrm{U}=5.3 \mathrm{eV}, \Delta E_{M}=-2.733 \mathrm{eV}\right)$. Interestingly, our refitted $\mathrm{U}$ and the $\Delta E_{M}$ terms are very close to the ones reported in the two methodology papers. 145 Recently, a revised correction scheme was proposed by the Materials Project group 10 and made available online. The results obtained using this scheme is labeled as MP-2020 ( $\mathrm{U}=5.3$ $\left.\mathrm{eV}, \Delta E_{M}=-2.256 \mathrm{eV}\right)$. We note the newer PBE_54 PAW data set is used in this work with Fe_pv for Fe, Li_sv for $\mathrm{Li}, \mathrm{O}$ for $\mathrm{O}$ and $\mathrm{S}$ for $\mathrm{S}$. The Materials Project uses the same pseudopotential choices, but from an older set named PBE.

\footnotetext{
${ }^{1}$ Using the data from the NIST-JANAF ${ }^{9}$ table gives very similar results.
} 
The reaction $\mathrm{FeS}+\mathrm{Li}_{2} \mathrm{~S} \longrightarrow \mathrm{FeO}+\mathrm{Li}_{2} \mathrm{O}$ can be used as a benchmark for the treatment of sulfides. Since it does not contain any elemental phase, the errors involved with them have no effect. Assuming that most of the DFT errors come from the treatment of the Fe, having a formation energy that is too negative means that the oxides are overly favored, and vice versa. As shown in Figure S1, using U for both oxides and sulfides makes $\mathrm{FeO}$ slightly favored (the All scheme), whereas if it is only applied to the oxides, the FeS is more favored (Oxide only). If one assumes that the $\Delta E_{M}$ correction for mixing GGA/GGA+U is sufficient for oxides, this implies that the standard GGA calculation overly stabilize FeS. The same behavior is found in oxides using plain GGA, where the errors from localized the $\mathrm{d}$ electrons are found to compensate some of that from the oxygen over-binding. "4 While applying $\mathrm{U}$ to only the oxides gives an error of similar magnitude here (Oxide only), we find that the inclusion of $\mathrm{U}$ is essential for the correctly predicting the relative stabilities of FeS polymorphs. Using standard PBE functional, the troilite phase $\left(P \overline{6} 2_{c}\right)$, a rare mineral and whose experimental formation energy is available, is predicted to be more than $200 \mathrm{meV} /$ per atom higher in energy compared to the tetragonal phase. 11 The data from Materials Project gives large negative errors for this particular reaction, the difference can be attributed to different calibrations and corrections. In fact, the data from the Materials Project appear to be incorrectly calibrated for reproducing the reaction energy of oxidizing $\mathrm{FeO}$ to $\mathrm{Fe}_{2} \mathrm{O}_{3}$ and $\mathrm{Fe}_{3} \mathrm{O}_{4}$ (shown in the Fitting $U$ for Fe of Figure S1), nor does it reproduce the binary formation energies of iron oxides (shown in the $G G A+U / G G A$ Mixing of Figure S1). The new material project correction (MP-2020) appears to be correctly calibrated to the binary oxide formation energies with a revised $\Delta E_{M}$, but it suffers from the same problem for the reaction energies of oxidizing $\mathrm{FeO}$, due to the inconsistent $U_{F e}$ calibration. On the other hand, both two sets of reaction energies are well reproduced by the 0xide only scheme, which is not a surprise, since it was to calibrated to reproduce them. The All scheme does not include the $\Delta E_{M}$ term and hence is not expect to give accurate formation energies for iron oxides, but we are only interested in the competition between the quaternary and binary 
phases here. Hence, the All scheme is most suitable for investigating the stabilities of the oxysulfides.

\section{Gibbs's free energy at finite temperature using machined learned descriptors}

The energies from the DFT calculations can only be used to access the thermodynamic stability at $0 \mathrm{~K}$. At finite temperatures, the synthesizability of a material depends on its Gibbs's free energy, but the incorporation of temperature effects is not trivial. The vibrational entropy may be accounted for using phonon density of states under the quasi-harmonic approximation, which can be very resource intensive. Recently, a machine learning model for estimating the Gibbs's free energy has been reported ${ }^{12}$ In that work, it was found that the Gibbs energy may be estimated to a good accuracy using the $0 \mathrm{~K}$ enthalpy given by DFT

plus an additional analytical term that is data mined using the sure independence screening and sparsifying operator (SISSO) method. This additional term only takes the composition of the material, the volume of the DFT relaxed structures, and the temperature as the input.

Applying this approach allows us to estimate the synthesizability at finite temperatures. In the Figure S2, the phases that are predicted to be close to the convex hull remain close at finite temperatures. Interestingly, the number of stable phases appears to increase with increasing temperature. This is reflected in both the computed distance to hull as well as the distance to hull formed by the known non-quaternary phases.

However, we note that these results under finite temperatures can have several caveats. First, the contribution of configurational entropy is not explicitly included, which could make disordered phases more favorable at high temperatures, and one example is the $\mathrm{Li}_{2} \mathrm{FeSO}$. Second, the physical descriptor was fitted using the Materials Project's data, which are computed using a similar but not identical pseudopotential set. In addition, the $\mathrm{U}$ for Fe d electron is $4 \mathrm{eV}$ here whereas the Materials Project uses $5.3 \mathrm{eV}$. This could result 
in inconsistencies in the both the energies of Fe-containing phases and their equilibrium volumes. Second, the training and testing set of the descriptor only includes the known experimental phases, whereas here a large set of newly predicted structures also exists. Many metastable phases that have higher volume per atom are found by our search, giving much reduced $G_{\text {SISSO }}^{\delta}$ term as a result of its volume dependency. In some cases, predicted phases that are more than $100 \mathrm{meV}$ above the hull at $0 \mathrm{~K}$ can be stabilized. Since such hypothetical structures are not included in the training set, result results are less reliable. As a result, we have to limit the phases considered in Figure S2 to those having the lowest DFT energy for each composition. Formation energies including free energy contributions under fixed volumes have been calculated using Phonopy, and compared with those predicted by SISSO as shown in Figure S3. Although a good agreement is found for $\mathrm{Li}_{4} \mathrm{Fe}_{3} \mathrm{~S}_{3} \mathrm{O}_{2}$, those for $\mathrm{Li}_{2} \mathrm{Fe}_{2} \mathrm{~S}_{2} \mathrm{O}$ and $\mathrm{Li}_{2} \mathrm{Fe}_{3} \mathrm{~S}_{2} \mathrm{O}_{2}$ have rather large deviations. Since the SISSO descriptor is fitted with constant pressure data, phonon calculations using the quasi harmonic approximation may provided data that are more applicable for comparison. Such validation study would be beyond the scope of this work. Finally, it should be noted that the descriptor itself is found to have mean absolute error (MAE) of $46 \mathrm{meV}$ as mentioned in the original paper, $\stackrel{12}{12}$ although some degrees of the error cancellations are expected as only four elements are included.

\section{Data availability}

The structure searching results, raw calculation data for phonon, NEB and hybrid functional calculations, an archive containing the provenance of all calculations for the AiiDA framework, and example notebooks for data analysis and plotting have been deposited into the Zenodo repository with open access. DOI: 10.5281/zenodo.4977231. 


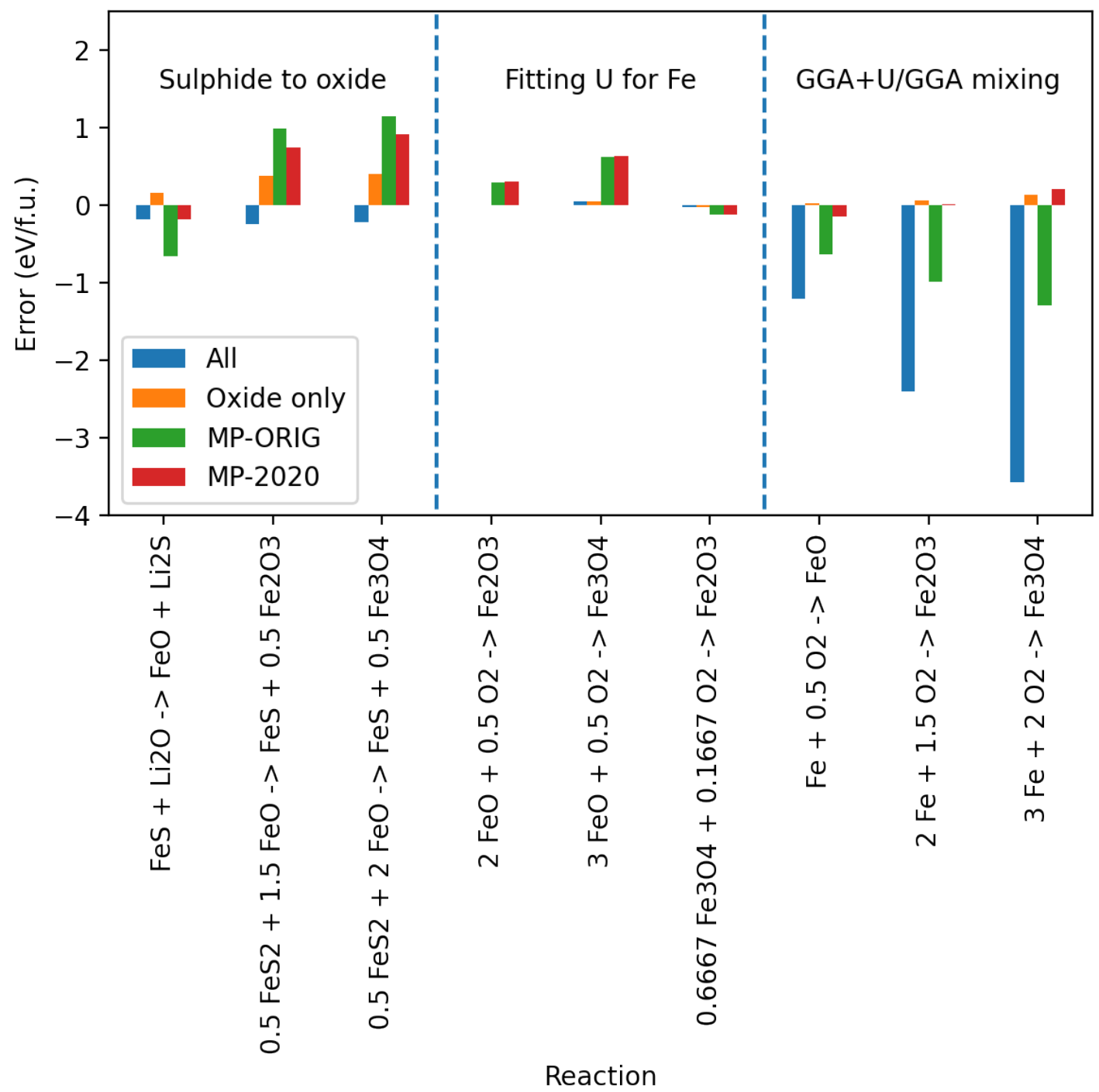

Fig. S1: Errors of the reaction energies using different schemes for treating Fe-containing oxides and sulfides with the Hubbard correction. The All scheme uses $U_{F e}=4.0 \mathrm{eV}$ for both oxide and sulfides. The Oxide only uses the $U_{F e}=4.0 \mathrm{eV}$ for oxides but not for sulfides, and the GGA+U energies are corrected by a $\Delta E_{M}$ term $(1.787 \mathrm{eV})$ so it can be mixed with the GGA calculations. The MP scheme uses the same methodology as the Oxide only, but the energies are obtained directly through the Materials Project API using their settings and corrections $\left(U_{F e}=5.3 \mathrm{eV}\right.$, and $\left.\Delta E_{M}=2.733 \mathrm{eV}\right)$. 

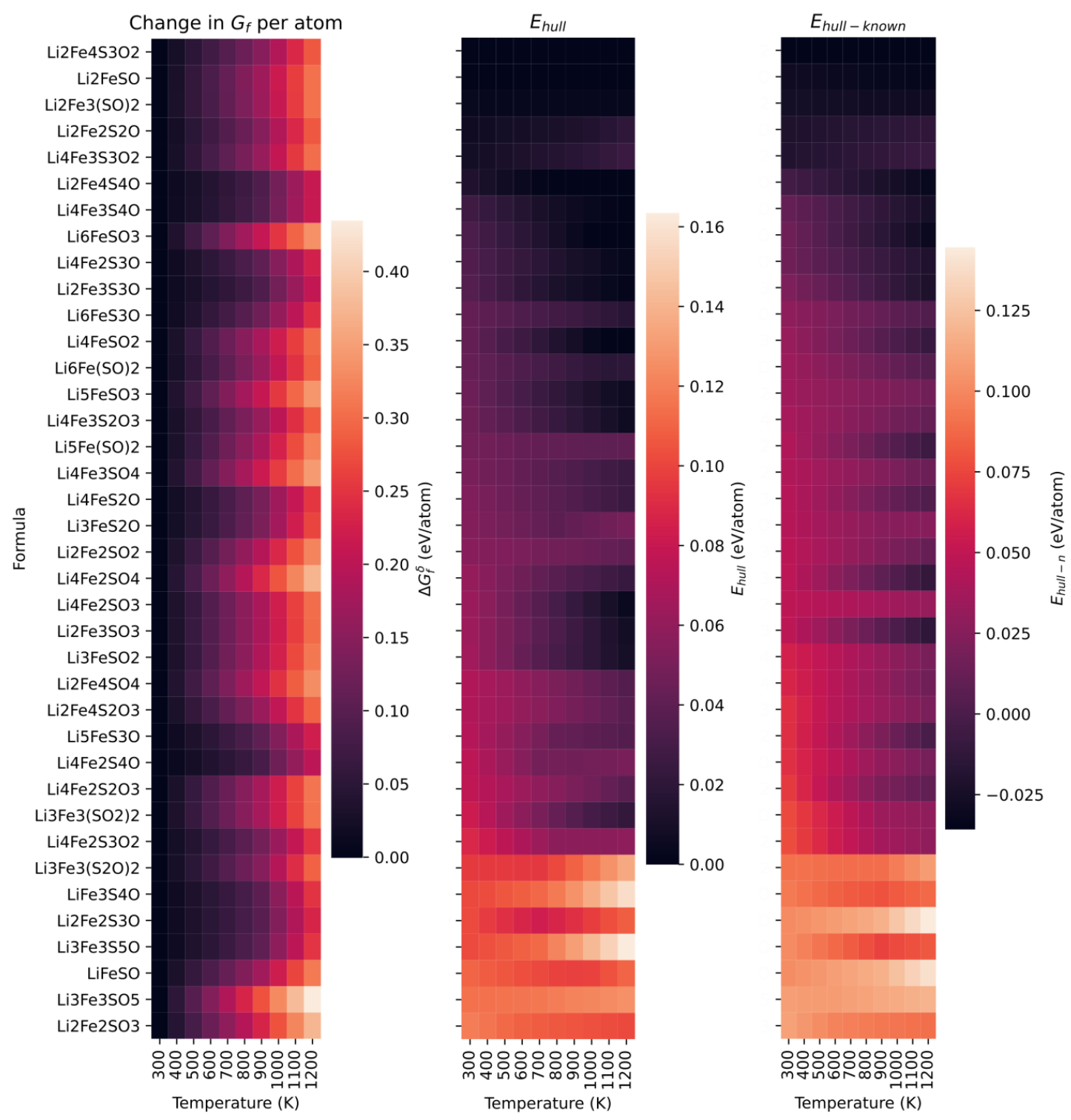

Fig. S2: Finite temperature stabilities predicted using the physical descriptors data mined by the SISSO method. ${ }^{12}$ It can be seen that the stabilities of most phases does not change much with temperature, but there are exceptions. 


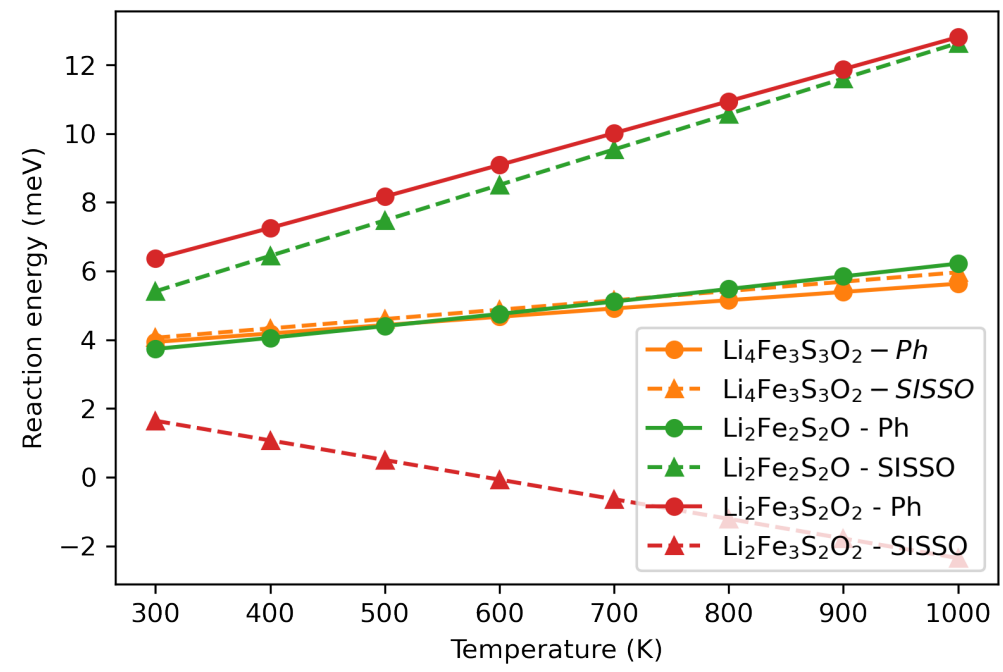

Fig. S3: Change in free energy calculated using phonopy compared to those estimated using the SISSO descriptor. 
a)

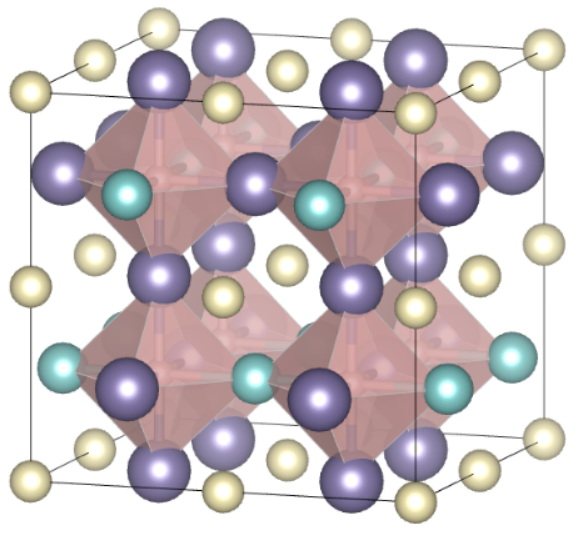

c)

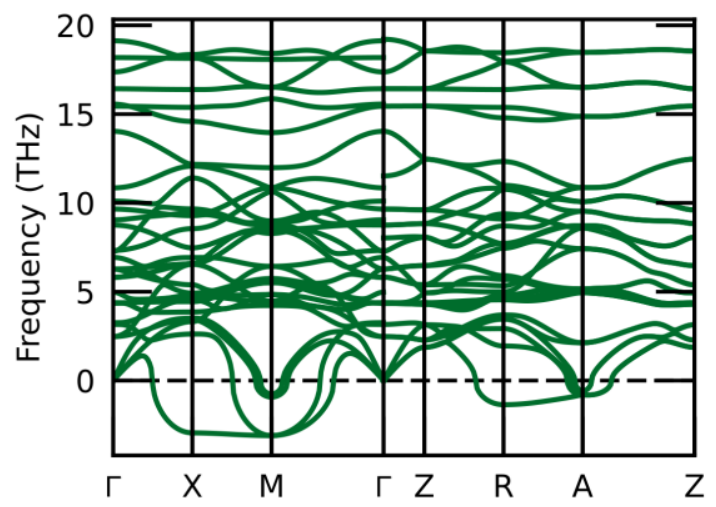

b)

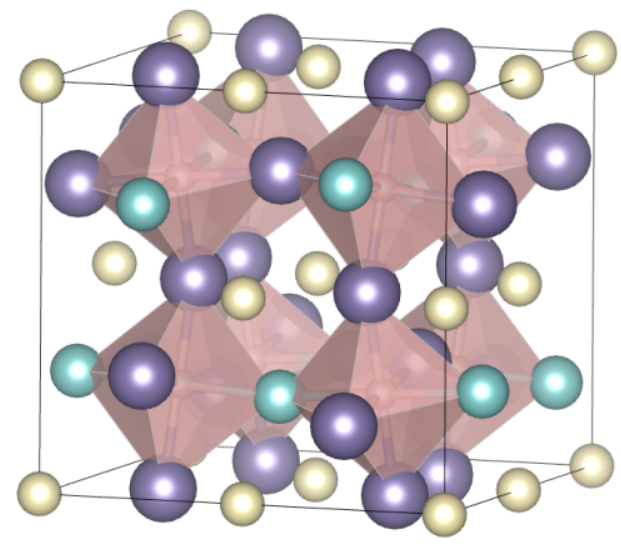

d)

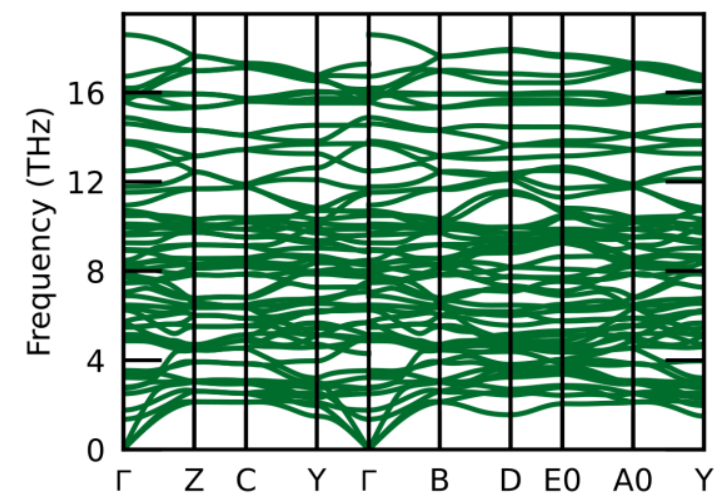

Fig. S4: (a) The ordered $\mathrm{Li}_{2} \mathrm{FeSO}$ structure reported previously.13 (b) The mode-pushed structure with $a^{-} b^{-} c^{0}$ tilted octahedra. (c) The phonon band structure of the ordered $\mathrm{Li}_{2} \mathrm{FeSO}$ with imaginary frequencies. (d) The phonon band structure of the mode-pushed structure without any imaginary frequency. Colour coding: cream-S, purple-Li, cyan-Fe, pink-O. 
a)

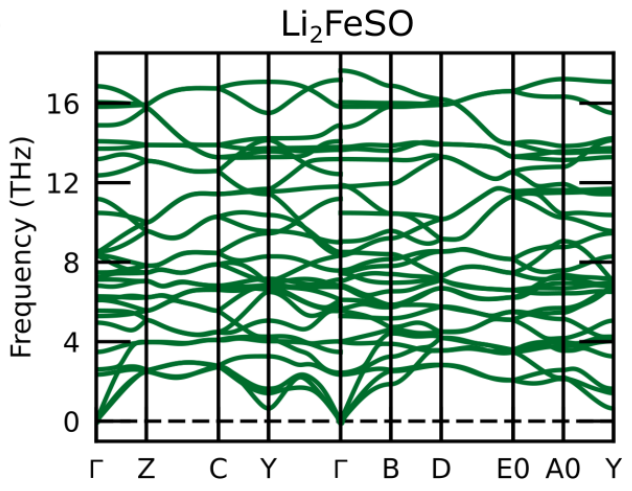

c)

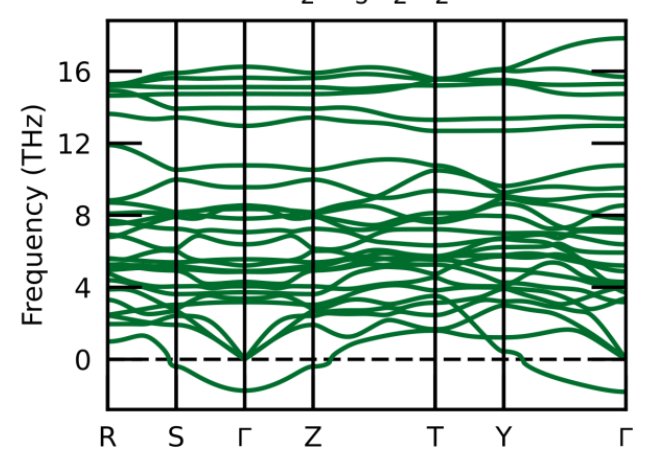

e)

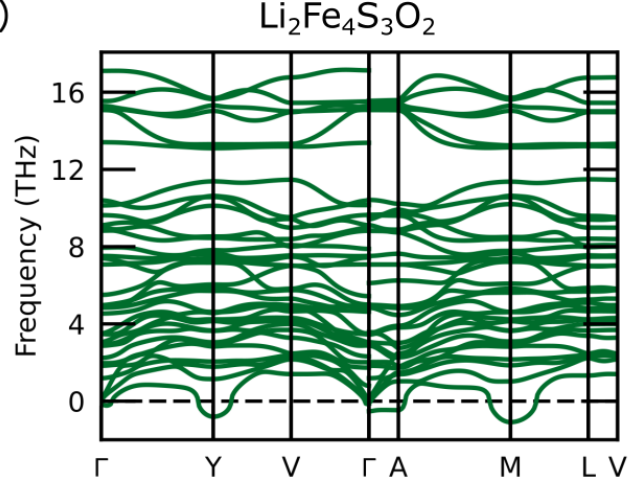

b) $\quad \mathrm{Li}_{4} \mathrm{Fe}_{3} \mathrm{~S}_{3} \mathrm{O}_{2}$

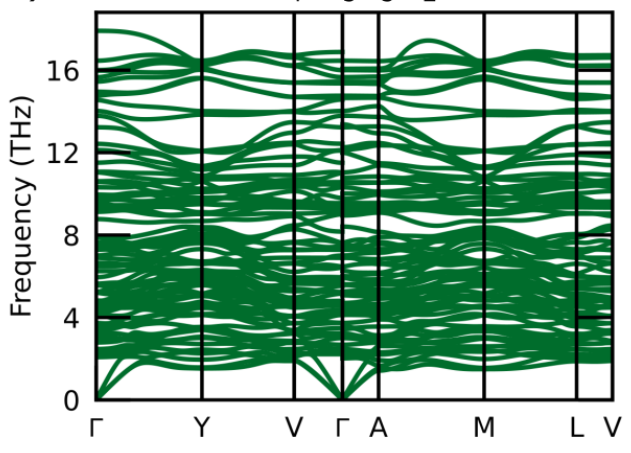

d) $\quad \mathrm{Li}_{2} \mathrm{Fe}_{3} \mathrm{~S}_{2} \mathrm{O}_{2}$ (pushed)

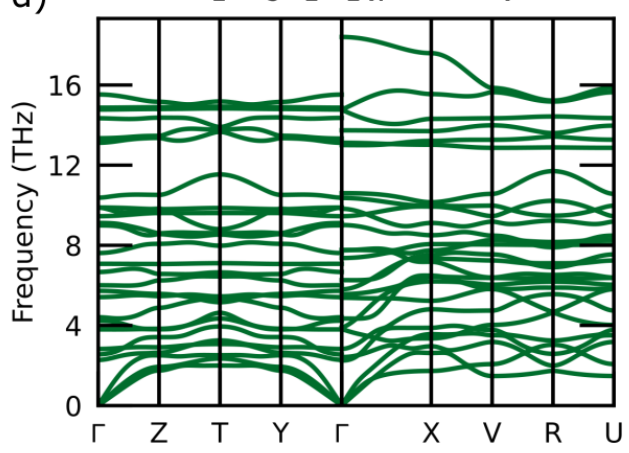

$\mathrm{Li}_{2} \mathrm{Fe}_{4} \mathrm{~S}_{3} \mathrm{O}_{2}$ (pushed)

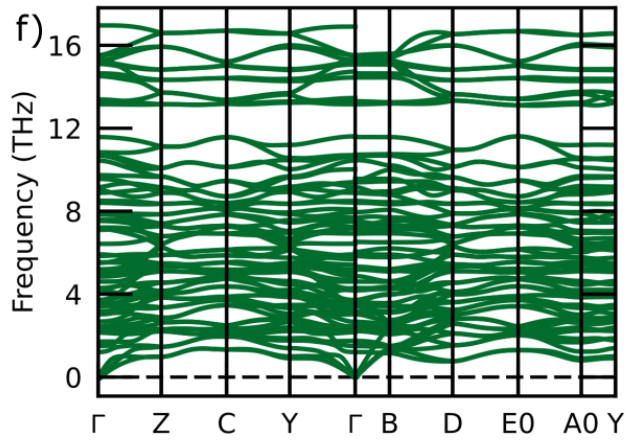

Fig. S5: Phonon dispersions calculated for (a) $\mathrm{Li}_{2} \mathrm{FeSO}$, (b) $\mathrm{Li}_{4} \mathrm{Fe}_{3} \mathrm{~S}_{3} \mathrm{O}_{2}$, (c,d) $\mathrm{Li}_{2} \mathrm{Fe}_{3} \mathrm{~S}_{2} \mathrm{O}_{2}$ and $(e, f) \mathrm{Li}_{2} \mathrm{Fe}_{4} \mathrm{~S}_{3} \mathrm{O}_{2}$ 

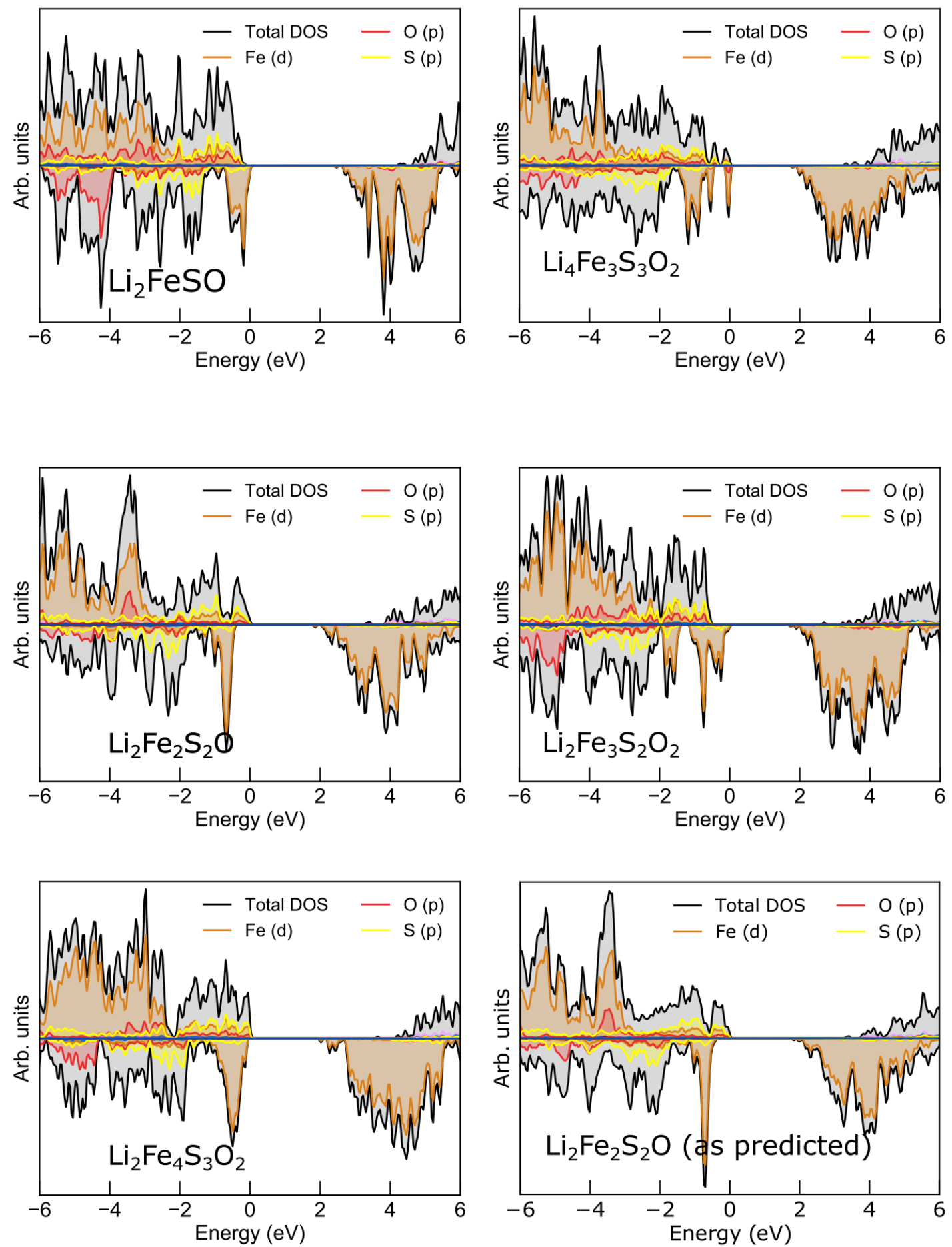

Fig. S6: Projected density of states for the predicted phases using HSE06 hybrid functional. The valance band tops are consisted of $\mathrm{Fe} d$ states, $\mathrm{O}$ p states and $\mathrm{S} p$ states, and the conduction band maximum is dominated by $\mathrm{Fe} d$ states. The band gaps of these materials are about $2 \mathrm{eV}$. The mode pushed $\mathrm{Li}_{2} \mathrm{Fe}_{2} \mathrm{~S}_{2} \mathrm{O}$ and the as-predicted $\mathrm{Li}_{2} \mathrm{Fe}_{2} \mathrm{~S}_{2} \mathrm{O}$ both have almost the same density of states. 
a)

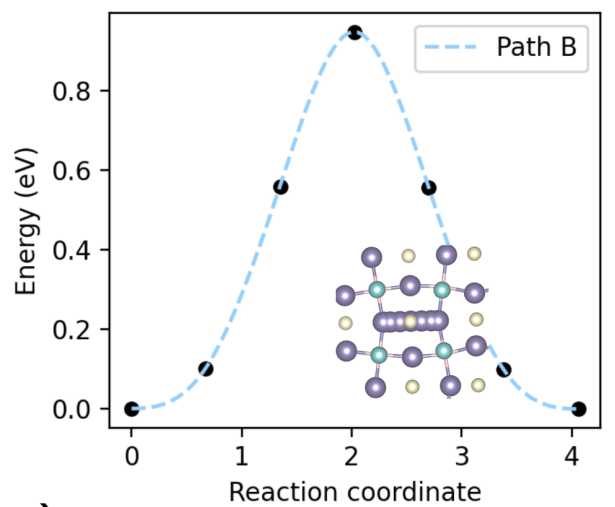

c)

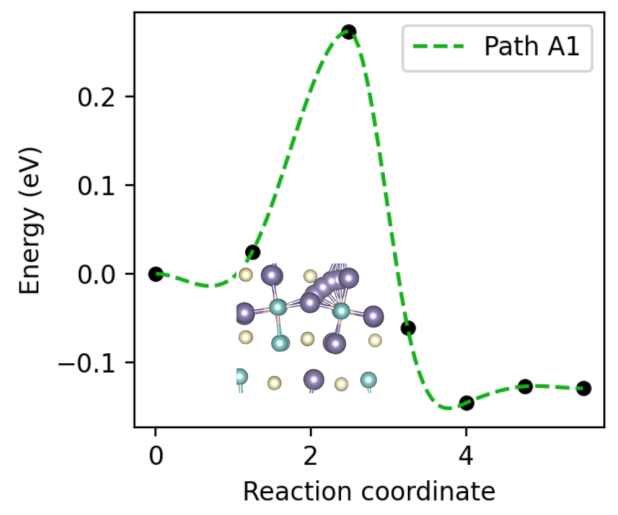

e)

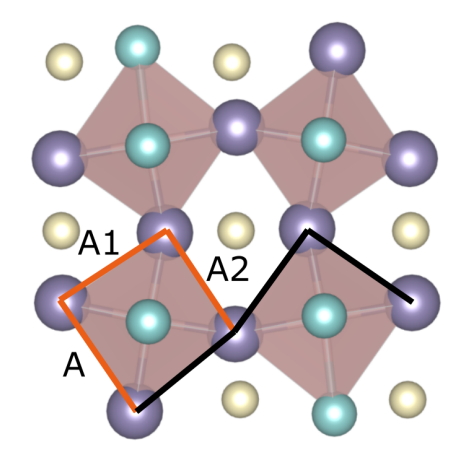

e) b)

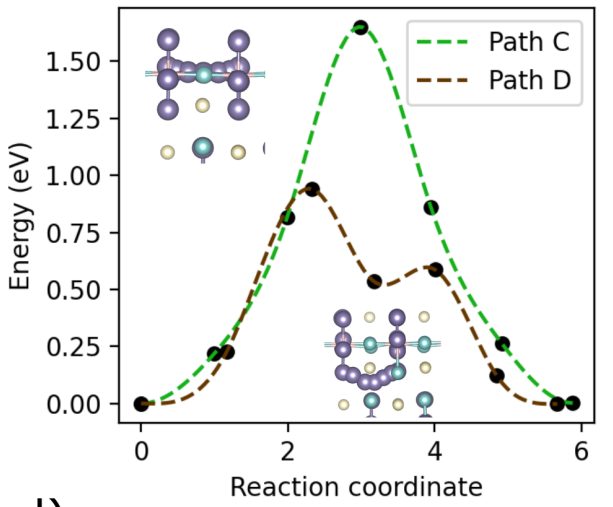

d)

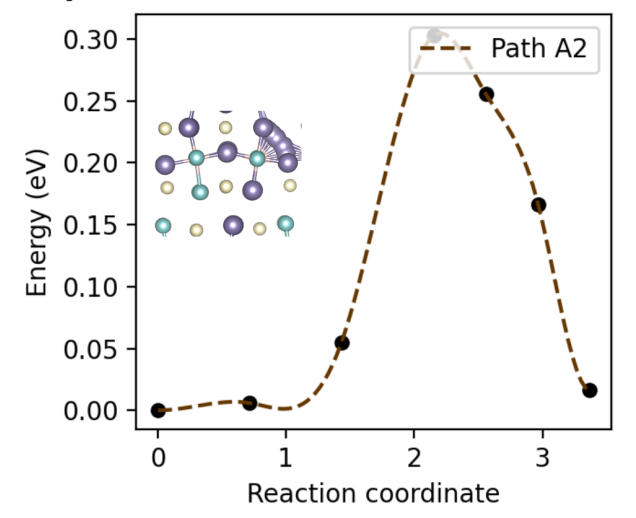




\section{References}

(1) Jones, R. O.; Gunnarsson, O. The Density Functional Formalism, Its Applications and Prospects. Reviews of Modern Physics 1989, 61, 689-746.

(2) Patton, D. C.; Porezag, D. V.; Pederson, M. R. Simplified Generalized-Gradient Approximation and Anharmonicity: Benchmark Calculations on Molecules. Physical Review $B$ 1997, 55, 7454-7459.

(3) Hammer, B.; Hansen, L. B.; Nørskov, J. K. Improved Adsorption Energetics within Density-Functional Theory Using Revised Perdew-Burke-Ernzerhof Functionals. Physical Review B 1999, 59, 7413-7421.

(4) Wang, L.; Maxisch, T.; Ceder, G. Oxidation Energies of Transition Metal Oxides within the GGA+U Framework. Physical Review B 2006, 73, 195107.

(5) Jain, A.; Hautier, G.; Ong, S. P.; Moore, C. J.; Fischer, C. C.; Persson, K. A.; Ceder, G. Formation Enthalpies by Mixing GGA and GGA + U Calculations. Physical Review B 2011, 84, 045115.

(6) Jain, A.; Ong, S. P.; Hautier, G.; Chen, W.; Richards, W. D.; Dacek, S.; Cholia, S.; Gunter, D.; Skinner, D.; Ceder, G.; Persson, K. A. Commentary: The Materials Project: A Materials Genome Approach to Accelerating Materials Innovation. APL Materials 2013, 1, 011002.

(7) Kirklin, S.; Saal, J. E.; Meredig, B.; Thompson, A.; Doak, J. W.; Aykol, M.; Rühl, S.; Wolverton, C. The Open Quantum Materials Database (OQMD): Assessing the Accuracy of DFT Formation Energies. npj Computational Materials 2015, 1, 1-15.

(8) Kubaschewski, O.; Kubaschewski, O.; Alcock, C. B. Metallurgical Thermochemistry; Elsevier Science \& Technology Books, 1979. 
(9) Allison, T. JANAF Thermochemical Tables, NIST Standard Reference Database 13. 1996.

(10) Wang, A.; Kingsbury, R.; McDermott, M.; Horton, M.; Jain, A.; Ong, S. P.; Dwaraknath, S.; Persson, K. A Framework for Quantifying Uncertainty in DFT Energy Corrections. 2021,

(11) Lai, X.; Zhang, H.; Wang, Y.; Wang, X.; Zhang, X.; Lin, J.; Huang, F. Observation of Superconductivity in Tetragonal FeS. Journal of the American Chemical Society 2015, 137, 10148-10151.

(12) Bartel, C. J.; Millican, S. L.; Deml, A. M.; Rumptz, J. R.; Tumas, W.; Weimer, A. W.; Lany, S.; Stevanović, V.; Musgrave, C. B.; Holder, A. M. Physical Descriptor for the Gibbs Energy of Inorganic Crystalline Solids and Temperature-Dependent Materials Chemistry. Nature Communications 2018, 9, 4168.

(13) Lu, Z.; Ciucci, F. Anti-Perovskite Cathodes for Lithium Batteries. Journal of Materials Chemistry A 2018, 6, 5185-5192. 